\title{
Comparison of Underwater and Overground Treadmill Walking to Improve Gait Pattern and Muscle Strength after Stroke
}

\author{
Si-Eun Park, PT, MS ${ }^{1)}$, Soon-Hee Kim, PT, PhD ${ }^{1)}$, Sang-Bin Lee, PT, PhD'2), \\ Ho-Jung An, PT, PhD ${ }^{3)}$, Wan-Suk Choi, PT, $\mathrm{PhD}^{4}$, Ok-Gon Moon, PT, $\mathrm{PhD}^{5}$, \\ Ji-Sung Kim, PT, $\mathrm{PhD}^{6}$, Hee-Joon Shin, PT, $\mathrm{PhD}^{7)}$, Yoo-Rim Choi, PT, $\mathrm{MS}^{8}$, \\ Kyoung-OK Min, PT, PhD ${ }^{1)}$ \\ 1) Department of Physical Therapy, Yongin University: 470 Samga-dong, Cheoin-gu, Yongin, Republic \\ of Korea.TEL: +82 31-8020-2771,E-mail:ptcountry@hanmail.net \\ 2) Department of Physical Therapy, Namseoul University \\ 3) Department of Physical Therapy, Daewon College \\ 4) Department of Physical Therapy, International University of Korea \\ 5) Department of Physical Therapy, Kunjang College \\ 6) Department of Physical Therapy, Suwon Women's College \\ 7) Department of Physical Therapy, Kyungwoon University \\ 8) Department of Physical Therapy, Daegu Science College
}

\begin{abstract}
Purpose] The objective of this study was to compare the effect of underwater and overground treadmill walking, which affect gait pattern and muscle strength in people with hemiparesis following a stroke. [Subjects] Twenty chronic stroke patients were included in this study. [Methods] The subjects were randomly allocated to one of two groups, the underwater treadmill walking (UTW) and overground treadmill walking (OTW) groups. The amount of weight the subjects exerted on the foot on the affected side, as well as the joint angle values and muscle strengths on the affected side, were measured. Intervention was performed 4 times per week, and each session lasted 30 minutes. [Results] It was shown that the weight exerted on both the entire foot and hindfoot increased more in the UTW group than in the OTW group. However, the weight put on the forefoot was not found to increase for either group. In terms of the joint angle values, the angle between the hip and the knee showed a greater increase in the UTW group than in the OTW group. In contrast, the ankle joint angle did not show significant change in either of the groups. Muscle strength showed a similar increase in both groups. [Conclusion] These results suggest that after a stroke underwater treadmill walking has a more positive effect on gait pattern than overground treadmill walking. Key words: Underwater treadmill, Stroke, Gait
\end{abstract}

(This article was submitted Sep. 29, 2011, and was accepted Jun. 11, 2012)

\section{INTRODUCTION}

Loss and impairment of walking ability is one of the major devastating outcomes for people who have suffered a stroke. While 25 percent of stroke survivors are never able to ambulate independently, in 50 percent of patients, walking impairments are still observed 3 months after a stroke ${ }^{1)}$. Thus, the restoration and improvement of walking ability in these subjects is one of the main goals during rehabilitation. The major causes of walking impairment are decreased muscle activity, difficulties with weight bearing and a reduced sense of proprioception ${ }^{2}$. During rehabilitation, therapists apply either a traditional functional approach to muscle strengthening or may use various neuro-facilitation techniques, such as the Brunnstrom technique, which uses synergistic movements, proprioceptive neuromuscular facilitation using spiral and diagonal movements, and neurodevelopmental(Bobath) therapy which utilizes reflex inhibitory movements ${ }^{3)}$. Richard et al. (2005) reported, however, that purpose-oriented walking methods, i.e., encouraging the patient to actually walk normally, were more effective than traditional training methods ${ }^{4}$. Treadmill training has been shown to have positive effects on walking ability and cardiovascular fitness, as well as to promote activation of the cerebellum, midbrain, and some parts of the cerebral cortex ${ }^{5}$.

Treadmill training cannot always be used in cases where the patient is no longer able to walk independently, for example, after a severe stroke. Therefore, underwater walking exercise has recently been proven as an effective way of practicing walking and exercise during training and rehabilitation ${ }^{6}$. The water environment provides a lower apparent body weight due to the buoyant force, providing more support to the body than on a normal treadmill ${ }^{7}$. However, it is difficult to compare walking in a swimming pool with overground treadmill walking. When walking in a 
swimming pool, it is difficult to maintain a constant speed, whereas this can be done simply on an overground treadmill. Therefore, an underwater treadmill apparatus was developed to control the speed of underwater walking. The underwater treadmill apparatus can be made to simulate various environments, and it is possible to adjust the temperature, the height of the water and walking speed.

The purpose of this study was to investigate the differences between underwater and overground treadmill walking for people recovering from a stroke. We evaluated body weight, joint angle and muscle strength on the affected side to compare the effects of rehabilitation treatment using underwater and overground treadmill walking.

\section{SUBJECTS AND METHODS}

The study subjects were selected from patients at the RUSK rehabilitation hospital (Seongnam, South Korea). Twenty patients who had suffered a stroke more than 6 months previously were included in this study. The participants were randomly allocated into underwater treadmill walking (UTW, n=10) and overground treadmill walking (OTW, $\mathrm{n}=10$ ) groups. All patients had to satisfy three main inclusion criteria: being able to walk $10 \mathrm{~m}$ independently without any walking aids, achieving a minimum of 22 points in the mini-mental state examination (MMSE), and being capable of understanding and following simple instructions. Patients with any neurological disorders other than a stroke and patients with orthopedic problems were excluded from the study. The aims and procedures involved were explained, and each patient signed a consent form before participating in the study. Table 1 outlines the general characteristics of the participants.

The trials consisted of underwater and overground treadmill walking. The subjects either walked on an underwater treadmill (Aqua Track, Woojin, Korea), immersed to the level of thoracic vertebrae 11 , or walked on an overground treadmill (HL-1100, Healthlife, Korea). The water temperature for the UTW group was maintained at $28^{\circ} \mathrm{C}-30{ }^{\circ} \mathrm{C}$; for the OTW group, the room temperature was maintained at $24{ }^{\circ} \mathrm{C}-26^{\circ} \mathrm{C}$. The speed of the treadmill was limited to a maximum velocity of $2-4 \mathrm{~m} / \mathrm{s}$ in both groups. The rehabilitation program consisted of 30 -minute walking sessions, four times per week for 6 weeks. If participants were experiencing shortness of breath or were feeling light headed or over exerted, they were allowed to take a break. The treadmills had emergency shutdown devices installed to ensure the safety of the participants.

The amount of weight patients exerted on each foot, their joint angles and their muscle strengths were recorded. Weight bearing ability on the affected side was assessed by using a SmartStep system. SmartStep system has (Andante Medical, White Plains, NY, USA), which is a mobile gait analysis system with biofeedback capabilities. The SmartStep system has pressure sensitive pneumatic insoles, which measures the weight exerted on the foot (including the entire foot, the forefoot and the hindfoot). Joint angles during walking (in the hip, knee, and ankle) were assessed using a digital video camera (Nikon D90, Japan). To calculate the joint
Table 1. General characteristics of the study participants

\begin{tabular}{lcc}
\hline & UTW & OTW \\
\hline Sex (male/female) & $5 / 5$ & $4 / 6$ \\
Age (years) & $51.8 \pm 14.4$ & $58.7 \pm 8.3$ \\
Weight (kg) & $61.8 \pm 9.3$ & $60.9 \pm 5.2$ \\
Height (cm) & $161.3 \pm 8.3$ & $159.3 \pm 10.1$ \\
Affected side (left/right) & $7 / 3$ & $5 / 5$ \\
Time after stroke (months) & $13.1 \pm 8.4$ & $12.5 \pm 8.4$ \\
$\begin{array}{l}\text { Type of stroke } \\
\text { (infarction/hemorrhage) }\end{array}$ & $6 / 4$ & $6 / 4$ \\
\hline
\end{tabular}

angle values, that is, displacements in the sagittal plane, a digital video camera was placed on the affected side of the subject. The joint angles of the hip and knee were measured by examining forward and backward motion along the gravity line. The axis of the hip joint was set as the greater trochanter, and the knee joint axis was set as the femoral epicondyle. The ankle joint angle was measured when the foot was on the ground, examining the angles of dorsiflexion and plantarflexion. Measurements were collected three times, and the results were averaged to obtain the final reading. The muscle strength of the lower extremities were assessed by using a Short Physical Performance Battery (SPPB). The SPPB comprises three components: a hierarchical balance task, a 4-meter walk, and five repetitive chair stands. The sum of the three components is used to calculate the SPPB score, which has a possible range of 0 to 12 . Higher scores reflect a better level of muscle strength. All participants in both groups were evaluated during, prior to, and 6 weeks after starting the intervention.

Data were analyzed using the SPSS for Windows version 12.0 software. A repeated measures of variance (ANOVA) was used to evaluate the changes in weight bearing ability, joint angles, and muscle strength over the study period within each group. An analysis of covariance (ANCOVA) was used to evaluate the differences in changes seen over the study period between the two groups. In all analyses, $p<0.05$ was considered significant.

\section{RESULTS}

All participants were able to perform underwater and overground treadmill walking.

Table 2 shows the weight exerted on the entire foot, hindfoot, and forefoot by the subjects on the affected side during walking. In the UTW group, the amount of body weight exerted on the entire foot increased by significantly more than that exerted on the entire foot in the OTW group throughout the course of the study $(p<0.05)$. In terms of the amount of weight put on the hindfoot, the UTW group also showed a greater increase throughout the study period than the OTW group $(\mathrm{p}<0.05)$. However, the change in body weight exerted on the forefoot showed no significant difference between the two groups.

Table 3 shows the joint angle values at the point of heel contact when the toe is raised from the ground during walking. 
Table 2. Body weight exerted on the entire foot, hindfoot, and forefoot (on the affected side)

\begin{tabular}{llllcc}
\hline & \multicolumn{2}{c}{ UTW } & & \multicolumn{2}{c}{ OTW } \\
\cline { 2 - 3 } \cline { 5 - 6 } & \multicolumn{1}{c}{ Before } & \multicolumn{1}{c}{ After } & & Before & After \\
\hline Entire foot $(\mathrm{kg})$ & $52.5 \pm 10.7$ & $55.5 \pm 11.2^{* *}$ & & $53.6 \pm 13.1$ & $54.1 \pm 12.6$ \\
Hindfoot $(\mathrm{kg})$ & $26.6 \pm 9.9$ & $28.6 \pm 10.7^{* *}$ & & $27.8 \pm 12.2$ & $28.4 \pm 13.1$ \\
Forefoot $(\mathrm{kg})$ & $33.5 \pm 9.7$ & $34.0 \pm 9.6$ & & $38.8 \pm 19.3$ & $39.5 \pm 18.8$ \\
\hline
\end{tabular}

Mean \pm standard deviation. Abbreviation: UTW, underwater treadmill walking; OTW, overground treadmill walking. ${ }^{*} \mathrm{p}<0.05,{ }^{* *} \mathrm{p}<0.01$

Table 3. Hip, knee, and ankle joint angle values at heel contact and toe-off

\begin{tabular}{lccccc}
\hline & \multicolumn{2}{c}{ UTW } & & \multicolumn{2}{c}{ OTW } \\
\cline { 2 - 3 } \cline { 5 - 6 } \cline { 5 - 6 } Before & After & & Before & After \\
\hline Heel contact & & & & & \\
Hip joint $\left({ }^{\circ}\right)$ & $17.5 \pm 5.6$ & $22.6 \pm 6.0^{* *}$ & & $17.9 \pm 5.6$ & $19.2 \pm 5.7^{*}$ \\
Knee joint $\left({ }^{\circ}\right)$ & $5.7 \pm 4.3$ & $8.1 \pm 4.0^{*}$ & & $6.1 \pm 3.7$ & $6.5 \pm 3.6$ \\
Ankle joint $\left({ }^{\circ}\right)$ & $2.0 \pm 3.5$ & $3.6 \pm 5.4$ & & $4.3 \pm 4.7$ & $4.7 \pm 4.8$ \\
\hline Toe-off & & & & & \\
Hip joint $\left({ }^{\circ}\right)$ & $-2.8 \pm 12.0$ & $0.7 \pm 12.3^{* *}$ & & $-3.7 \pm 13.6$ & $-3.1 \pm 13.8$ \\
Knee joint $\left({ }^{\circ}\right)$ & $29.8 \pm 7.4$ & $33.6 \pm 5.0^{*}$ & & $29.3 \pm 7.5$ & $30.1 \pm 7.1$ \\
Ankle joint $\left({ }^{\circ}\right)$ & $40.7 \pm 6.5$ & $41.6 \pm 5.1$ & & $39.3 \pm 12.0$ & $39.2 \pm 10.2$ \\
\hline
\end{tabular}

Mean \pm standard deviation. Abbreviation: UTW, underwater treadmill walking; OTW, overground treadmill walking. ${ }^{*} \mathrm{p}<0.05,{ }^{* *} \mathrm{p}<0.01$

Upon heel contact, the hip joint was significantly more flexed in the UTW group than in the OTW group $(\mathrm{p}<0.01)$. The knee joint was also significantly more extended in the UTW group than in the OTW group $(\mathrm{p}<0.05)$. However, the ankle joint angles showed no significant difference over time within groups or between groups.

At the point at which the toe was raised from the ground (hereafter referred to as "toe-off"), the hip joint was significantly more extended in the UTW subjects than in the OTW subjects $(\mathrm{p}<0.05)$. The knee joint was also significantly more flexed in the UTW group than in the OTW group $(\mathrm{p}<0.05)$. The angle made by the ankle joint, however, showed no significant within-group or between-group differences.

Table 4 shows the lower extremity muscle strengths recorded by SPPB. The SPPB score significantly improved after rehabilitation in both groups. No significant difference was observed between the two groups.

\section{DISCUSSION}

The main objective of this study was to investigate the differences in the effects of underwater and overground treadmill walking rehabilitation in people who had had a stroke. The body weight exerted on the foot, joint angles, and muscle strength data were evaluated in order to compare treadmill walking under the two different environmental conditions. The results of this study revealed that both groups showed improved muscle strength after the study period. However, UTW had a more positive effect than OTW in terms of the amount of body weight patients could exert on the foot and the joint angles exhibited during walking.
Table 4. Change in lower extremity strength measured using SPPB

\begin{tabular}{cccccc}
\hline & \multicolumn{2}{c}{ UTW } & & \multicolumn{2}{c}{ OTW } \\
\cline { 2 - 3 } \cline { 5 - 6 } & Before & After & & Before & After \\
\hline $\begin{array}{l}\text { SPPB } \\
\text { (score) }\end{array}$ & $7.0 \pm 2.7$ & $8.6 \pm 2.5^{* *}$ & & $6.9 \pm 1.1$ & $8.5 \pm 1.4^{* *}$ \\
\hline
\end{tabular}

Mean \pm standard deviation. Abbreviation: UTW, underwater treadmill walking; OTW, overground treadmill walking. ${ }^{* *} \mathrm{p}<0.01$

The amount of body weight exerted on the entire and hindfoot showed a significant difference between groups, such that in the UTW group, there was a significant improvement in the weight bearing ability of subjects at the end of the study period; however, the OTW group did not show a significant level of improvement. One of the main reasons for this might be that the segmental postures adopted during underwater walking are close to the body's center of gravity. In a previous study ${ }^{8}$, water-based exercise allowed subjects to maintain the body's gravity line naturally, which may have meant that subjects adopted a more neutral position while walking. In addition, Kaneda et al. (2008) reported improved dynamic balance after waterbased exercise ${ }^{9)}$. This may have caused the apparent body weight exerted on the entire foot and hindfoot to increase on the affected side during underwater treadmill walking. In contrast, body weight put on the forefoot did not show a significant difference between groups. Neither group showed improvement in the amount of weight put on the forefoot and no significant difference was apparent in the ankle joint angles of both groups. 
In this study, joint angles in the hip, knee, and ankle were measured at the time of heel contact and when lifting the toe from the ground. Upon heel contact, the angles of the hip and knee joint were much larger in the UTW group than in the OTW group. Hung et al. (2008) reported that the disparity in the angle of the hip joint on the affected and unaffected sides in people with stroke was lessened after underwater training ${ }^{10)}$. Kaneda et al. (2008) detected higher muscle activity level in the rectus femoris during underwater walking than overground walking9), further giving support to the findings of this study. However, the joint angles in the ankle at the point of heel contact showed no significant difference both within each group after rehabilitation or between groups. In a previous study ${ }^{11}$, greater voluntary contraction of the tibialis anterior muscle was observed during underwater walking than during overground walking, which may be attributed to the ankle joint angle. This result is inconsistent with the findings of our study, and this may be due to the difference in time periods over which the two studies were performed. In this case, we may assume that a study period of 6 weeks was not long enough for changes in ankle joint angle to take effect.

Similar results were found at the point of lifting the toe from the ground. There were significant changes in the hip and knee joint angles in the UTW group but not in the OTW group, whereas there were no significant changes in the joint angle of the knee in either group. These results suggest that UTW is an effective method of rehabilitation for improving movement in the hip and knee joints after a stroke.

The SPPB was performed to investigate muscle strength. The results indicated significant improvement in muscle strength for both groups after rehabilitation; however, there was no significant difference between groups in terms of the amount of improvement seen. Harris et al. (2002) reported that muscle activity levels in the quadriceps femoris during treadmill walking were higher than during overground walking ${ }^{12)}$, suggesting that treadmill walking has a positive effect on muscle strength under both environmental conditions (underwater and over ground).

\section{REFERENCES}

1) Wade DT, Wood VA, Heller A: Walking after stroke measurement and recovery over the first three months. Scand J Rehabil Med, 1987, 19: 25-30. [Medline]

2) Burdett RG, Borello-France D: Blatchly, Poptter C: Gait comparison of subjects with hemiplegia walking unbraced, with ankle-foot orthosis, and with Airstrrup brace. Phys Ther, 1998, 68: 1197-1203.

3) Brandstater ME, Basmajian JV: Sensorimotor neurophysiology and the basis of neurofacilitation therapeutic techniques. Stroke Rehabil, 1987, 1: 109-183.

4) Macko RF, Ivey FM, Forrester LW, et al.: Treadmill exercise rehabilitation improves ambulatiry function and cardiovascular fitness in patients with chronic stroke. Stroke, 2005, 36: 2206-2211. [Medline] [CrossRef]

5) Duncan PW: Stroke disability. Phys Ther, 1994, 74: 399-407. [Medline]

6) Wadell K, Sundelin G, Henriksson-Larsen K, et al.: High intensity physical group training in water an effective training modality for patients with COPD. Respir Med, 2004, 98: 428-438. [Medline] [CrossRef]

7) Barela AM, Stolf SF: Marcos Duarte: Biomechanical characteristics of adults walking in shallow water and on land. J Electromyogr Kinesiol, 2006, 16: 250-256. [Medline] [CrossRef]

8) Kim HM, Woo SY, Kim GW: Gait comparison of the stroke patients ground \& aqua-rehabilitation program for one year after 12 weeks aquarehabilitation program. J Adapted Phys Act Exerc, 2007, 15: 245-259.

9) Kaneda K, Sato D, Wakabayashi H, et al.: Comparison of different waterexercise programs' effects on balance ability in elderly people. J Aging Phys Act, 2008a, 16: 281-292.

10) Hung SB, Cho HG, Jung TW: The effect of underwater exercises program with buoyancy appliances on hemiplegies' gait of patients with hemiplegia. Journal of Special Physical Education. Yongin Univ, 2008, 6: 81-96.

11) Kato $T$, Sugagima $Y$, Koeda M, et al.: Electromyogram activity of leg muscles during different types of underwater walking. Advertise Exerc Sports Physiol, 2002, 8: 39-44.

12) Harris-Love ML, Forrester LW, Macko RF, et al.: Hemiparetic gait parameters in overground versus treadmill walking. Neurorehabil Neural Repair, 2001, 15: 105-112. [Medline] [CrossRef] 